\title{
The vibration reliability analysis of micro aircraft engine rotor Feng Ding ${ }^{1, a}$, Fengwei Qin ${ }^{2, b}$ \\ Xi'an Technological University, NO.2 Xuefu Middle Road, Xi'an, Shaanxi, P.R. China \\ aemail: 136790921@qq.com, bemail: 13572022495@163.com
}

Keywords: Rotor of aircraft engine; Vibration reliability; Proportional hazards model; Fminsearch function

\begin{abstract}
The rotor system is an important part of the aircraft engine, its running state directly influences the whole engine working condition. Therefore, the reliability analysis of rotor system is particularly important. Paper combines the vibration characteristics of the equipment operation condition with the traditional reliability analysis theory, put forward the proportional hazards model for vibration reliability analysis, and using MATLAB fminsearch function in parameter estimation, calculate the equipment reliability at present, then apply it to the micro aircraft engine rotor in the reliability analysis. Practice has proved that the method can achieve the equipment reliability effectively.
\end{abstract}

\section{Introduction}

Reliability refers to the probability that the products complete the required function within the prescribed time and conditions, reliability analysis of mechanical equipment can master the running state, and find fault in advance ${ }^{[1]}$. The traditional reliability analysis is mainly based on the theory of strength and stress distribution, the premise is the strength and stress distribution types are known, which is based on the theory of probability and statistics. The determination of strength and stress distribution type is the key that must use enough sample data, to determine the stress distribution must determine the failure mode of parts, and analyse the stress unit cell, calculate stress component and principal stress. Finally, synthesize stress distribution with algebraic method, moment method and monte-carlo method ${ }^{[2]}$. Determine the strength distribution must determine the strength criterion in the first place, through fixing nominal strength and determining each parameter of the strength formula to determine the strength distribution. So, make sure the distribution must design a large number of tests to obtain the relevant parameters, it can not combine with running information of equipments, it is show troublesome in the practical engineering application ${ }^{[3]}$.

Vibration signal often contains rich equipment running status information, through the analysis of vibration signal can get certain understanding of equipment running status, vibration reliability is based on the traditional theory of reliability, which combines with mechanical equipment running status vibration information to analyse reliability, it can effectively master the equipment running status $^{[4]}$. Paper put forward proportional hazards model that based on the equipment running status under the vibration characteristics, the model set up the relationship of vibration characteristic value (RMS, Kurtosis value and Peak, etc.) of the equipment operation condition and traditional reliability theory, then estimate the parameters of model, get the current reliability to master running status of equipment. 


\section{Weibull proportional hazards model and parameter estimation \\ Weibull proportional hazards model}

Proportional failure rate model is derived from proportional hazards models, put forward by COX of the British statistician in 1972 originally, which is mainly used in biology, medicine, etc firstly, along with the reliability is finding wider and wider application in the engineering, weibull proportional hazards model is well applied. The basic form of proportion failure rate model as following ${ }^{[5]}$;

$$
h(\mathrm{t}: \mathrm{Z})=h_{0}(\mathrm{t}) * \exp (\gamma * \mathrm{Z})
$$

$h_{0}(\mathrm{t})$ : baseline risk function.

$Z$ : the value of influence factor (covariate), the equipment vibration characteristics: RMS, Kurosis etc.

$\gamma$ : regression coefficient, stands for the effect that factors variable to failure rate.

Weibull distribution is derived from the Swedish physicist W.W eibull when analyzing strength of materials, weibull distribution has a strong ability of fitting for various types of experimental data, and weibull distribution can adapt the three failure phase of the bathtub curve, so get a wide range of applications ${ }^{[6]}$.

Weibull distribution has three parameters, $m, \eta, \gamma$, " $m$ "is the shape parameter, “ $\eta$ "is the scale parameter, “ $\gamma$ ”is the location parameter. In general, failure may occur starting from the product use, so in practice, " $\gamma$ "often is zero, namely two parameter weibull distribution ${ }^{[7]}$;

$$
f(\mathrm{t})=\frac{\beta}{\eta} *\left(\frac{t}{\eta}\right)^{\beta-1} * \mathrm{e}^{-(\mathrm{t} / \eta)^{\beta}} \quad t \geq 0, m, \eta>0
$$

failure rate function of two parameters weibull distribution is;

$$
h(\mathrm{t})=\frac{m}{\eta} *\left(\frac{t}{\eta}\right)^{m-1}
$$

substitute into the proportional failure rate model;

$$
h(\mathrm{t}: \mathrm{Z})=\frac{m}{\eta}\left(\frac{t}{\eta}\right)^{m-1} * \exp \left(\mathrm{r}^{*} \mathrm{Z}\right)
$$

get reliability function of weibull proportion model;

$$
R(t: Z)=\exp (-H(t: Z))=\exp \left[-\int_{0}^{t} \frac{\beta}{\eta} *\left(\frac{t}{\eta}\right)^{\beta-1} * \exp \left(\gamma^{*} Z\right) d t\right]
$$

\section{Parameters Estimation}

Using the proportional hazards model, estimate the parameters of “ $\beta$ ”, ” $\eta$ ” and " $\gamma$ ” first of all. There are three main kinds of parameters estimation method: moment estimation, the least square method and maximum likelihood estimate method, maximum likelihood estimation method is not only suitable for linear model parameter estimation, but also be applied to nonlinear parameter model to estimate. The thinking of maximum likelihood estimation is the probability of the biggest 
events is most likely to occur. Paper uses the maximum likelihood estimation method to estimate the parameters of the model .

Suppose the all samples are " $n$ ", failure samples are " $r$ ", the rest of the " $n-r$ " are truncated samples, obtain the likelihood function is ${ }^{[8]}$ :

$$
L=\prod_{i=1}^{r} f\left(t_{i}\right) * \prod_{r+1}^{n} R\left(t_{i}\right)
$$

Substitute the two parameters weibull proportional hazards probability density function and reliability function into the likelihood function;

$$
L(\beta, \eta, \gamma)=\prod_{i=1}^{r}\left[\frac{\beta}{\eta}\left(\frac{t_{i}}{\eta}\right)^{\beta-1} * \exp \left(\gamma * \mathrm{Z}\left(\mathrm{t}_{i}\right)\right)\right] * \prod_{j=1}^{n}\left[\exp \left(-\left(\frac{t_{j}}{\eta}\right)^{\beta}\right) * \exp \left(\gamma * \mathrm{Z}\left(\mathrm{t}_{i}\right)\right]\right.
$$

Logarithmic likelihood function is;

$$
\operatorname{In}[\mathrm{L}]=r * \operatorname{In}\left(\frac{\beta}{\eta}\right)+\sum_{i=1}^{r} \operatorname{In}\left(\frac{t_{i}}{\eta}\right)^{\beta-1}+\sum_{i=1}^{r} \gamma * Z\left(\mathrm{t}_{i}\right)-\sum_{j=1}^{n}\left[\left(\frac{t_{j}}{\eta}\right)^{\beta} * \exp \left(\gamma * Z\left(\mathrm{t}_{i}\right)\right)\right]
$$

The partial derivatives of “ $\beta$ ”, “ $\eta$ ” and” $\gamma$ ”;

$$
\begin{aligned}
& \frac{\partial \operatorname{In} L}{\partial \beta}=\frac{r}{\beta}+\sum_{i=1}^{r} \operatorname{In}\left(\frac{t_{i}}{\eta}\right)-\sum_{j=1}^{n}\left(\left(\frac{t_{i}}{\eta}\right)^{\beta} * \operatorname{In}\left(\frac{t_{j}}{\eta}\right) * \exp \left(\gamma * \mathrm{Z}\left(\mathrm{t}_{j}\right)\right)\right) \\
& \frac{\partial \operatorname{In} L}{\partial \eta}=-\frac{r}{\eta}+\sum_{j=1}^{n}\left(\frac{\beta}{\eta} *\left(\frac{t_{j}}{\eta}\right)^{\beta} * \exp \left(\gamma * \mathrm{Z}\left(\mathrm{t}_{j}\right)\right)\right) \\
& \frac{\partial \operatorname{In} L}{\gamma}=\sum_{i=1}^{r} \mathrm{Z}\left(\mathrm{t}_{i}\right)-\sum_{j=1}^{n}\left(\mathrm{Z}\left(\mathrm{t}_{j}\right) *\left(\frac{t_{j}}{\eta}\right)^{\beta} * \exp \left(\gamma * \mathrm{Z}\left(\mathrm{t}_{j}\right)\right)\right)
\end{aligned}
$$

Set above all equations are 0 , so get a set of nonlinear equations, fminsearch function of MATLAB is used to solve the multidimensional unconstrained unlinear optimization problem. The equations can be solved by using fminsearch functions through setting the initial value.

\section{The application of Weibull proportional hazards model in micro aero-engine rotor system}

In this paper, two groups of micro aero-engine rotor vibration data is collected, and the length is 10400000, the sampling frequency is $50 \mathrm{KHZ}$. The first group of rotor vibration signals starting from the engine has been running $205 \mathrm{~h}$, speed remains the same, then the second group of rotor vibration signal after a period of time, calculates the RMS, Kurtosis value of vibration per 16000 data as a set, it is concluded the 650 groups of RMS and Kurtosis value respectively, the 650 groups of RMS and Kurtosis value as the two collaborators variables of proportional hazards model, as shown in figure 1, 2 and 3, 4; 


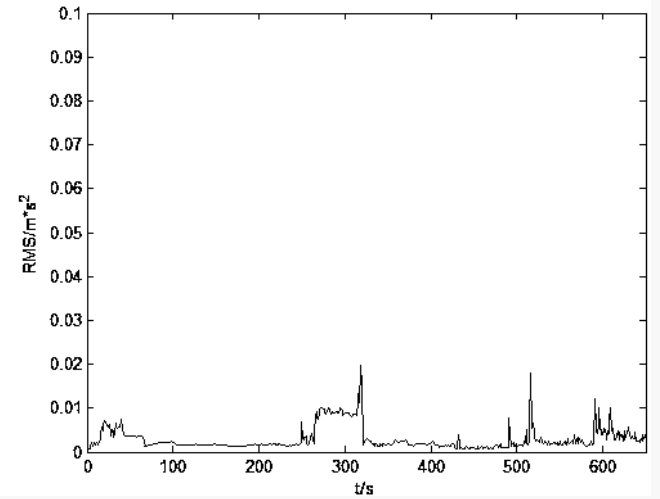

Fig.1 The RMS of first group

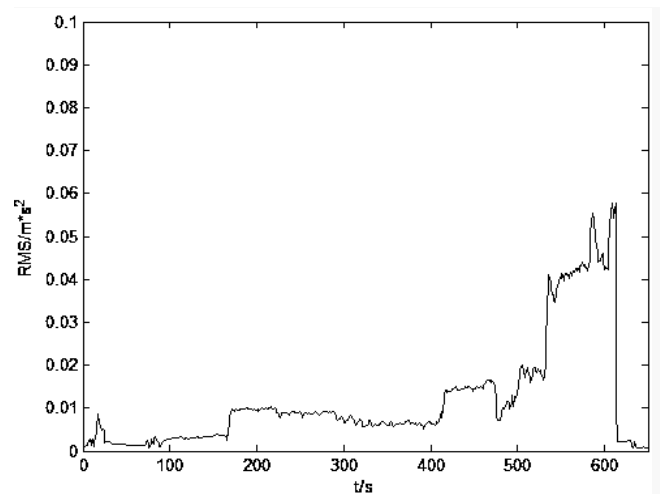

Fig.3 The RMS of second group

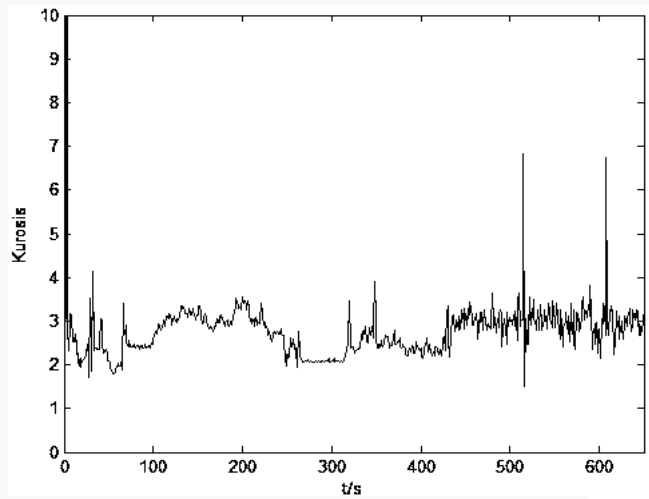

Fig.2 The Kurtosis of first group

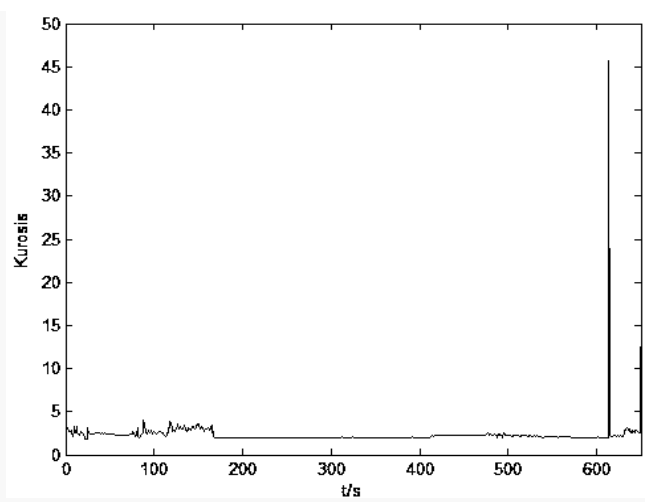

Fig.4 The Kurtosis of second group

Substitute the RMS and Kurtosis value calculated into the proportional hazards model, estimate the parameters using fminsearch function, the results of parameters estimation in table 1;

Then substitute the parameters into the weibull proportional reliability function, calculate the reliability of micro aero-engine rotor respectively, shown in figure 5, and 6;

Table.1 The result of parameters estimation

\begin{tabular}{ccccc}
\hline Rotor & $\beta$ & $\eta$ & $\gamma_{1}$ & $\gamma_{2}$ \\
\hline First group & 1.10 & 4773.6 & 0.30 & 0.20 \\
Second group & 1.00 & 5000.2 & 0.51 & 0.53 \\
\hline
\end{tabular}




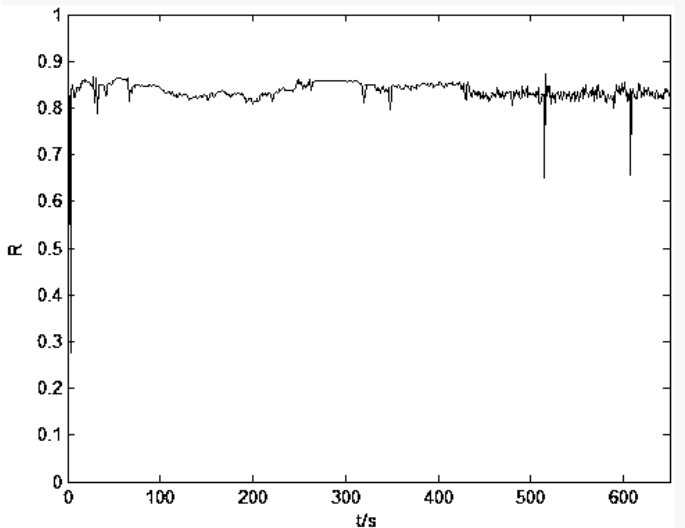

Fig.5 The reliability of first group

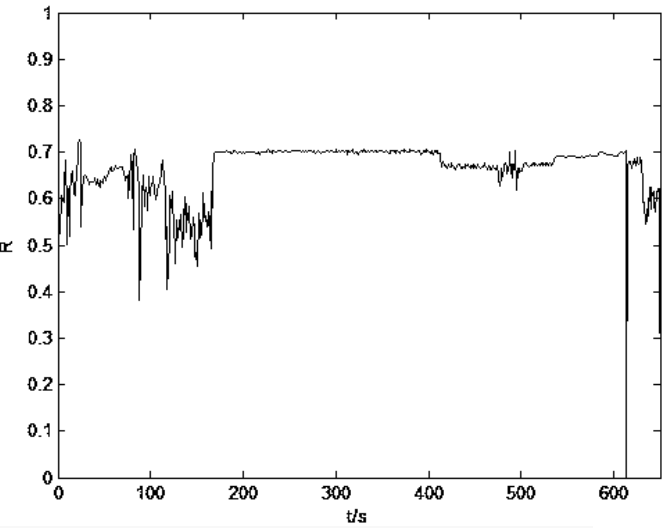

Fig.6 The reliability of second group

From the figure above, the reliability of first group data is 0.85 or so, corresponding to the rotor condition that engine has been running for $205 \mathrm{~h}$. The reliability of second group is about 0.65 , reliability decline compared with the first group, reflect the trend of reliability change with time accurately, it consistent with the actual situation. Therefore, the proportional hazards model can analyze the reliability of micro aero-engine rotor effectively.

\section{Conclusion}

Reliability analysis of mechanical equipment is a big difficulty in the current reliability engineering, the traditional reliability analysis method needs a large number of equipment failure data without combining with the current operation information. Weibull proportional hazards model is an effective method in many reliability analysis methods, through the vibration characteristics of the equipment current information, combines with the traditional reliability theory, achieve the reliability analysis of equipment effectively, it has the significant practical application value.

\section{Acknowledgment}

This research was financially supported by the National Science Foundation of China under grant 51275374 and the Fund Project of Science \& Technology on Reliability \& Environmental Engineering Key Laboratory.

\section{References}

[1]Feng Ding,Zhengjia He. Operational Reliability Evaluation of Machinery Considering Component Damaged Severity[J].Journal of xi 'an jiaotong university,2010,44(1):36-39.

[2]Weixin Liu.Mechanical reliability design[M].Tsinghua university press.1996.

[3]Zhengjia He,Hongrui Cao. Developments and Thoughts on Operational Reliability Assessment of Mechanical Equipment[J].Journal of MechanicalEngineering,2014,50:178-184.

[4]Gaigai Cai,Xuefeng Chen.Operaing Relibility Assesment By Equipment Response Condition Information[J].Journal of xi 'an jiaotong university.2012,46(1):108-115.

[5]Liao Haitao, Zhao Wenbiao, GUO Huairui. Predicting remaining useful life of Symposium[J],2006,127-132.

[6]Ding Feng,Zhengjia He.Reliability Assessment Based on Equipment Condition Vibration Feature Using Proportional Hazards Model[J].Chinese Journal of Mechanical 
Engineering,2009,45(18):55-60.

[7]Jhy-Ping Jhang.The Optimal Maintenance Policy Proportion for the Repairable Warranty Equipment Using Decresing-Failure-Rate-Reduction Method.[J]IEEE.2010

[8]Jardine A K S, Lin D, Banjevic D. A review on machinery diagnostics and prognostics implementing condition-based maintenance[J].Mechanical Systems and Signal Processing, 2006, 20(7): 1483-1510. 